\title{
Amplification and attenuation of noisy expression by export processes
}

\author{
Madeline Smith ${ }^{1}$, Mohammad Soltani ${ }^{2}$, Rahul Kulkarni ${ }^{3}$ Abhyudai Singh ${ }^{4}$ 粌
}

\begin{abstract}
Inside mammalian cells, single genes are known to be transcribed in stochastic bursts leading to the synthesis of nuclear RNAs that are subsequently exported to the cytoplasm to create mRNAs. We systematically characterize the role of export processes in shaping the extent of random fluctuations (i.e. noise) in the mRNA level of a given gene. Using the method of Partitioning of Poisson arrivals, we derive an exact analytical expression for the noise in mRNA level assuming that the nuclear retention time of each RNA is an independent and identically distributed random variable following an arbitrary distribution. These results confirm recent experimental/theoretical findings that decreasing the nuclear export rate buffers the noise in mRNA level, and counterintuitively, decreasing the noise in the nuclear retention time enhances the noise in the mRNA level. Next, we further generalize the model to consider a dynamic extrinsic disturbance that affects the nuclear-tocytoplasm export. Our results show that noise in the mRNA level varies nonmonotonically with the disturbance timescale. More specifically, high- and lowfrequency external disturbances have little impact on the mRNA noise level, while noise is amplified at intermediate frequencies. In summary, our results systematically uncover how the coupling of bursty transcription with nuclear export can both attenuate or amplify noise in mRNA levels depending on the nuclear retention time distribution and the presence of extrinsic fluctuations.
\end{abstract}

\section{Introduction}

Gene expression is the process by which a cell converts information stored within its DNA into functional gene products. The level of gene product has been found to exhibit considerable variation within genetically identical cells of the same population. This is referred to as gene expression noise [1,2]. Variability can cause deleterious effects

\footnotetext{
${ }^{* 1}$ M. Smith is with the Department of Electrical and Computer Engineering, University of Delaware, Newark, DE USA 19716. smithmadeudel .edu

${ }^{\dagger} \mathrm{M}$. Soltani is with the Department of Electrical and Computer Engineering, University of Delaware, Newark, DE USA 19716. msoltani@udel.edu

$\ddagger^{3}$ R. Kulkarni is with the Department of Physics, University of Massachusetts Boston, Boston, MA USA 02125. Rahul.Kulkarni@umb. edu

${ }^{\S} \mathrm{A}$. Singh is with the Department of Electrical and Computer Engineering, Biomedical Engineering, Mathematical Sciences, Center for Bioinformatics and Computational Biology, University of Delaware, Newark, DE USA 19716. absingh@udel. edu
} 
within the cell. For example, housekeeping genes encode types of proteins that carry out necessary cellular functions. The prevalence of noise can be detrimental as the level of these essential proteins must be tightly maintained to ensure proper functioning [3-5]. The study of gene expression noise and the mechanisms that drive it are an immense area of interest [6-11].

Gene expression noise is driven by intrinsic and extrinsic sources [12-15]. We consider intrinsic noise as variation that arises due to the inherently stochastic biochemical reactions involved in gene expression, such as transcription and translation. The key players involved in these reactions, like RNA and mRNA, exist at low copy numbers, thus amplifying variation. Moreover, extrinsic noise is the variation due to cell-tocell factors, such as fluctuations in the cell environment, cell growth, and cell cycle stage [16-19]. In this analysis, we first consider a standard gene expression model featuring intrinsic variations arising from bursty expression, then consider effects on overall noise in the presence of an extrinsic disturbance.

Within the events of gene expression, we are specifically interested in the RNA export process. RNA is a molecule that exports genetic information from DNA stored in the cell nucleus into the cytoplasm as mRNA; a key step in gene expression. The RNA is transported through nuclear pore complexes mediated by nuclear export receptors [20]. Studies reveal that variations in export, such as changes in pathway machinery or export factors, can lead to disease [21]. Therefore, we aim to provide insight into the role of export processes in shaping downstream gene expression noise.

The contribution of nuclear export processes in modulating stochastic gene expression is studied by considering how noise is affected when the (1) export timing is varied, (2) export process includes a time delay, and (3) export occurs in the presence of an extrinsic disturbance. The paper is structured as follows. In section II, we introduce the gene expression model formulation. In section III, we use Partitioning of Poisson Arrivals (PPA) to obtain a general formula for describing noise in cytoplasmic mRNA in the absence of extrinsic noise. The formula is used to characterize the effects on mRNA noise for different RNA nuclear retention time distributions. We further present results when nuclear RNA export includes extrinsic disturbances. Finally, conclusions are presented in section IV.

\section{Model Formulation}

We introduce a stochastic gene expression model with variability due to intrinsic sources. In this setup, gene expression begins in the cell nucleus where we consider a constitutive gene (i.e., the gene is always transcriptionally active) with no feedback regulation. The gene promoter produces bursts of nuclear RNA transcripts, modeled as a bursty birth-death process [22-28]. The bursting events occur at a rate $k_{x}$ as per a Poisson process to create $B$ number of RNA transcripts, where $B$ is a discrete random variable drawn from the probability distribution

$$
\text { Probability }\{B=j\}=\alpha_{j}, j \in\{1,2,3, \ldots\} .
$$

RNA transcripts subsequently undergo export from the nucleus into the cytoplasm. Export of a single RNA molecule occurs at rate $t_{x}$ to create a messenger RNA (mRNA) 


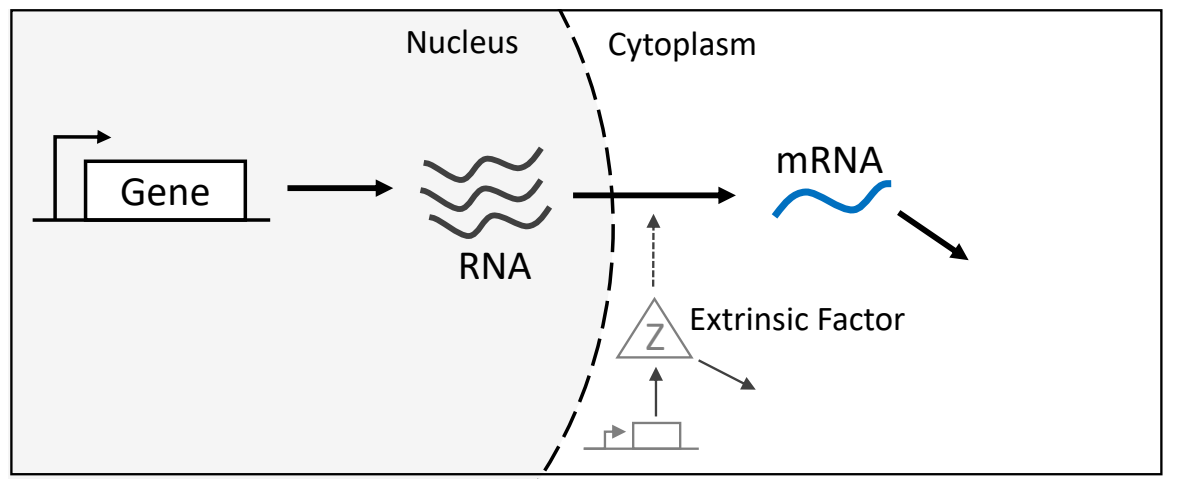

Figure 1: Gene expression model of the nuclear export process in the presence of an extrinsic disturbance. Within the cell nucleus, the gene synthesizes bursts of RNA transcripts that are subsequently exported to the cytoplasm to create mRNA. The cytoplasmic mRNA then decays. An extrinsic factor $Z$ is modeled as a bursty birth-death process and affects the nuclear export process.

transcript in the cytoplasm. The mRNA decays with rate $\gamma_{x}$. Prior work has shown that in the limit of rapid export $\left(t_{x} \rightarrow \infty\right)$ the steady-state noise in the cytoplasmic mRNA population counts, as quantified by the Fano Factor $F F$ (the variance dived by the mean) is given by

$$
F F_{\text {rapid }}=\frac{\left\langle B^{2}\right\rangle+\langle B\rangle}{2\langle B\rangle},
$$

where \langle\rangle is the expected value operation [29].

\section{Results}

\subsection{General Formula Describing Noise in Gene Expression}

In the section, we present a general result for the extent of fluctuations in mRNA population counts assuming the time spent by an individual RNA in the nucleus is an independent and identically distributed random variable following an arbitrary probability distribution $h$. Using the Partitioning of Poisson arrivals approach developed in [30], we obtain the following expression for the Fano factor at time $t$

$$
F F=1+\frac{\left\langle B^{2}\right\rangle-\langle B\rangle}{\langle B\rangle} \frac{\int_{0}^{t}\left[\int_{s}^{t} h\left(s_{1}-s\right) e^{-\gamma_{x}\left(t-s_{1}\right)} d s_{1}\right]^{2} d s}{\int_{0}^{t}\left[\int_{s}^{t} h\left(s_{1}-s\right) e^{-\gamma_{x}\left(t-s_{1}\right)} d s_{1}\right] d s}
$$

starting with zero number of RNAs in the system at $t=0$ (details of the proof are in Appendix A). Note that in the limit of non-bursty RNA production $(B=1$ with probability one) one obtains a Poisson distribution of mRNA counts with $F F=1$.

We can use the general result (3) to explore the effects of diverse nuclear retention time distributions. First, we consider a delta distribution where each RNA spends a 
fixed time in the nucleus. Replacing $h\left(s_{1}-s\right)$ in (3) with $\delta\left(s_{1}-s\right)$ and further simplifications results in the exact same noise

$$
F F_{\text {delta }}=\frac{\left\langle B^{2}\right\rangle+\langle B\rangle}{2\langle B\rangle} .
$$

as in the case of fast RNA export (2).

Next considering a exponentially-distributed nuclear retention time with mean $1 / t_{x}$ we substitute $h\left(s_{1}-s\right)=t_{x} e^{-t_{x}\left(s_{1}-s\right)}$ in (3) to obtain

$$
F F_{\text {exponential }}=1+\frac{\left\langle B^{2}\right\rangle-\langle B\rangle}{\langle B\rangle} \frac{t_{x}}{2\left(\gamma_{x}+t_{x}\right)} .
$$

Here the noise level $F F_{\text {exponential }}<F F_{\text {delta }}$ is smaller as compared to the delta-distributed case (4) with desynchronized RNA export events leading to effective noise buffering, As expected, (5) reduces to (2) in the limit $t_{x} \rightarrow \infty$. Furthermore, $F F_{\text {exponential }} \rightarrow 1$ as $t_{x} \rightarrow 0$ which is consistent with prior experimental/theoretical findings that slow nuclear export attenuate noise in mRNA levels [31-36].

Table 1: Stochastic gene expression model of a one-step nuclear export process. Here $x_{0}(t), x_{1}(t)$ and $z(t)$ are integer-valued stochastic processes representing the level of the nuclear RNA, cytoplasmic mRNA and extrinsic factor $Z$ at time $t$, respectively. The events describing the production of $Z$ in bursts and its decay are described under

\begin{tabular}{|c|c|c|}
\hline Event & $\begin{array}{c}\text { Change in } \\
\text { Population Count }\end{array}$ & $\begin{array}{c}\text { Probability Event will } \\
\text { Occur in }(t, t+d t)\end{array}$ \\
\hline Transcriptional Bursting & $x_{0}(t) \rightarrow x_{0}(t)+j$ & $k_{x} \alpha_{j} d t, j=1,2, .$. \\
\hline Nuclear Export & $\begin{array}{l}x_{0}(t) \rightarrow x_{0}(t)-1 \\
x_{1}(t) \rightarrow x_{1}(t)+1\end{array}$ & $\frac{z(t) t_{x}}{\langle z\rangle} x_{0}(t) d t$ \\
\hline mRNA Degradation & $x_{1}(t) \rightarrow x_{1}(t)-1$ & $\gamma_{x} x_{1}(t) d t$ \\
\hline Extrinsic Noise Production & $\begin{array}{c}z(t) \rightarrow z(t)+i \\
\text { (t) }\end{array}$ & $\begin{array}{c}k_{z} \alpha_{i} d t, i=1,2, . . \\
\end{array}$ \\
\hline Extrinsic Noise Degradation & $z(t) \rightarrow z(t)-1$ & $\gamma_{z} z(t) d t$ \\
\hline
\end{tabular}
the the dashed line.

\subsection{Extrinsic Fluctuations in Nuclear Export}

Next, we consider some form of extrinsic fluctuations in the export process by considering an extrinsic factor $Z$. The stochastic dynamics of this factor is modeled analogously 
to the RNA production earlier, where the factor is synthesized at a Poisson rate $k_{z}$ in random burst of size $B_{z}$. The factor decays with rate $\gamma_{z}$ that is related to the timescale of fluctuations in $Z$ levels. Using (2), the steady-state coefficient of variation $C V_{Z}$ in the level of $Z$ is given by

$$
C V_{Z}^{2}=\frac{\left\langle B_{z}^{2}\right\rangle+\left\langle B_{z}\right\rangle}{\left\langle B_{z}\right\rangle \overline{\langle z\rangle}}
$$

where $\overline{\langle z\rangle}=\left\langle B_{z}\right\rangle k_{z} / \gamma_{z}$ is the steady-state mean level of the extrinsic factor.

\subsubsection{Extrinsic Fluctuations in One-Step Delay}

Extrinsic fluctuations are first incorporated into the one-step transport process, as described in Fig. 1, where the RNA export rate is now proportional to the level of $Z$. For a fixed level $z(t)$ of $Z$, the nuclear retention time is exponentially-distributed with mean $\overline{\langle z\rangle} /\left(z t_{x}\right)$. Thus, if $Z$ happens to be higher than average at a given time point, then all nuclear RNA with be exported at a much faster rate. RNA export slows down as fluctuations in $Z$ revert back to average levels. The overall stochastic model is described in Table 1 with stochastic events that "fire" with some probabilities in the next infinitesimal time step, and when they occur, populations counts are reset by integer amounts.

We use the Method of Moments (as explained in Appendix B) to obtain the following expression for the steady-state Fano factor of mRNA levels

$$
F F_{n=1}=\overbrace{1+\frac{\left\langle B^{2}\right\rangle-\langle B\rangle}{\langle B\rangle} \frac{t_{x}}{2\left(\gamma_{x}+t_{x}\right)}}^{\text {Fano factor of one-step delay }}+C V_{Z}^{2} \frac{\langle B\rangle k_{x} \gamma_{x} \gamma_{z}}{\left(\gamma_{x}+t_{x}\right)\left(\gamma_{x}+\gamma_{z}\right)\left(\gamma_{z}+t_{x}\right)},
$$

where the first term is as derived in (5) in the absence of $Z$, and the second term is the contributions from the extrinsic disturbance.

The Fano factor reveals how an extrinsic disturbance in the export process would affect downstream noise in cytoplasmic mRNA. We first consider an extremely stable extrinsic noise factor $Z$ by taking the limit of $\gamma_{z} \rightarrow 0$

$$
\lim _{\gamma_{z} \rightarrow 0} F F_{n=1}=1+\frac{\left\langle B^{2}\right\rangle-\langle B\rangle}{\langle B\rangle} \frac{t_{x}}{2\left(\gamma_{x}+t_{x}\right)}=F F_{\text {exponential }}
$$

and the Fano factor simplifies to (5) - the Fano factor in the absence of any extrinsic factor. Thus, static fluctuations in $Z$ are not transmitted downstream to the mRNA level.

We next consider how average RNA transport time affects mRNA noise, and find that the mRNA Fano factor is not always buffered by increasing RNA transport time as before. Results in Fig. 2 depict noise buffering at increased RNA transport time when the extrinsic factor is not incorporated. However, when the extrinsic factor is included, the mRNA Fano factor exhibits non-monotonic behavior, where the Fano factor initially decreases before increasing, in relation to the average RNA transport time. The extent of the increased Fano factor at higher average RNA transport times is additionally influenced by the variability and the mean level of the extrinsic factor. This indicates that in the presence of extrinsic fluctuations, short average RNA transport 


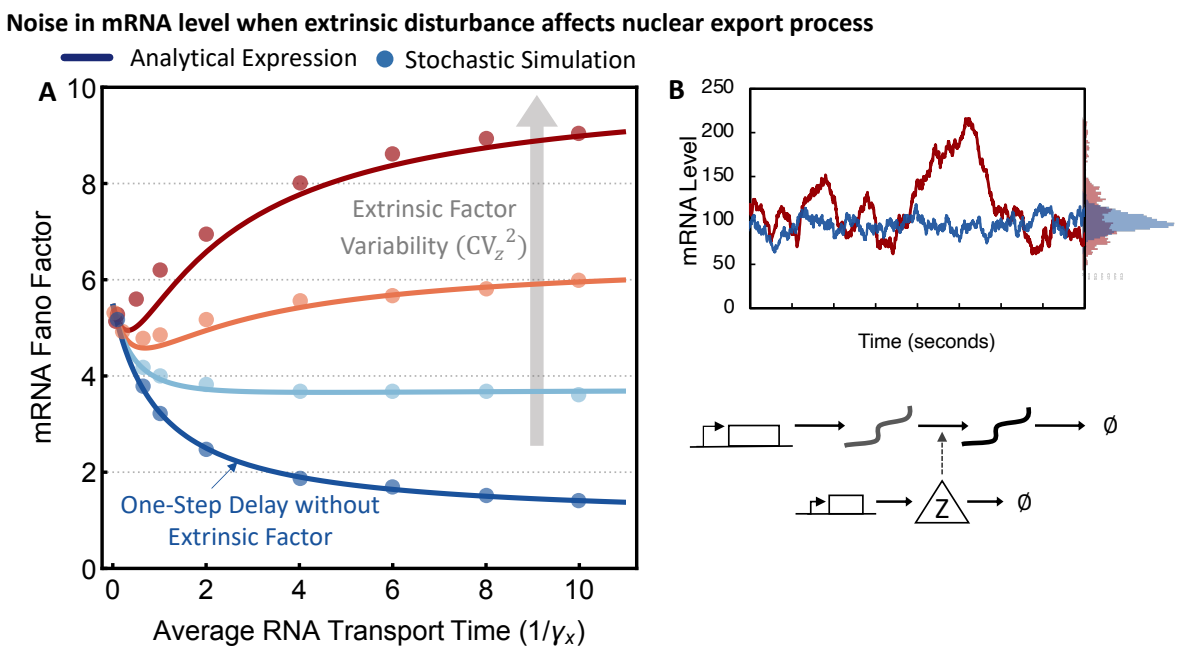

Figure 2: (A) Noise in mRNA level for a one-step transport process is plotted as a function of increasing transport time, normalized by the mRNA decay rate when extrinsic disturbances affect the transport process. The extrinsic factor, modeled by species $Z$, is altered to become more or less noisy. The dark blue line denotes the mRNA Fano factor without the extrinsic disturbance. The effect of increasing variability in extrinsic factor level, quantified by the extrinsic factor level coefficient of variation squared, is depicted by the light blue, orange, and red lines, with the values of $C V_{Z}^{2}=0.06,0.1$, and 0.6. Monte Carlo Simulations (SSA) results are denoted by circle markers and validate the analytical formulas. (B) Example traces of mRNA level fluctuations with time. The red line denotes mRNA level in the presence of an extrinsic factor with $C V_{Z}^{2}=0.6$ while the blue line denotes mRNA level in the presence of an extrinsic factor with $C V_{Z}^{2}=0.06$. The remaining parameters have the following values: $\langle B\rangle=10, k_{x}=10$, $\left\langle B_{z}\right\rangle=10, \gamma_{z}=1, t_{x}=1$. 


\section{Noise in mRNA level when extrinsic disturbance affects nuclear export process}

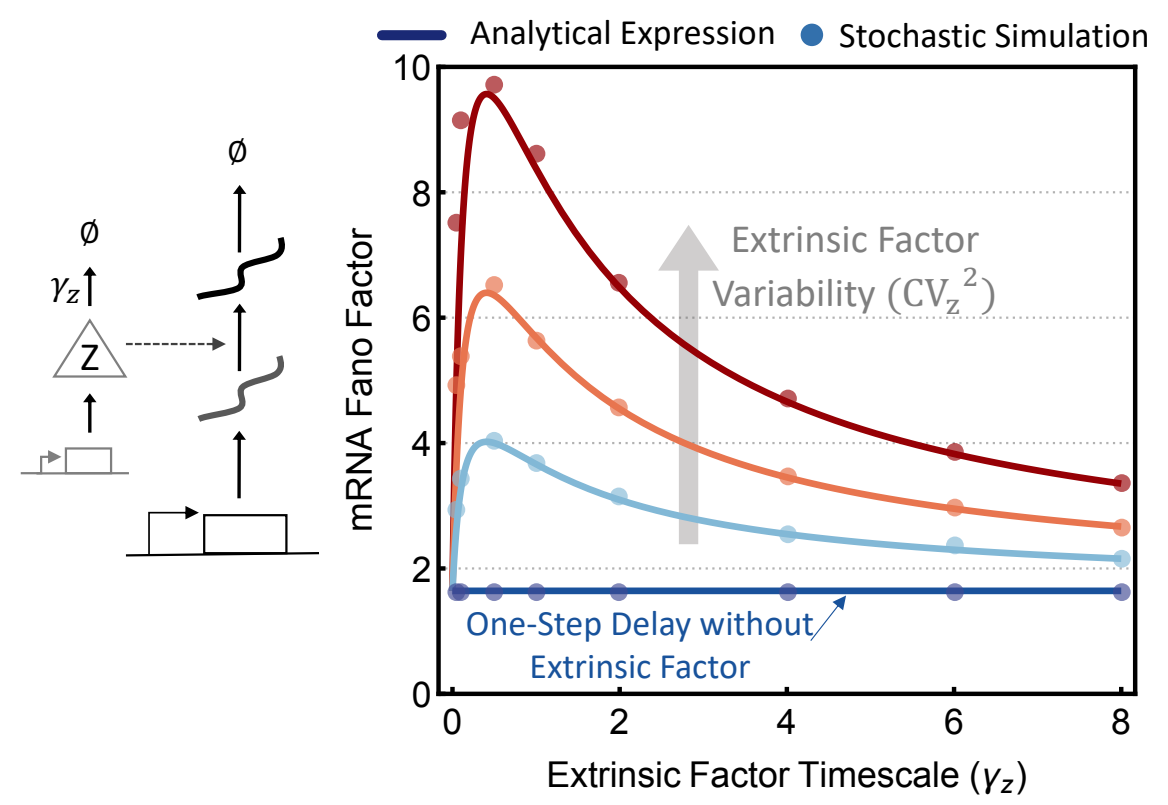

Figure 3: Cytoplasmic mRNA level noise as a function of extrinsic factor timescale. For each case, the mean level of extrinsic factor is held constant. mRNA Fano factor is plotted for increasing variation in the extrinsic factor level, quantified by its coefficient of variation squared, taking on the following values: $C V_{Z}^{2}=0.06,0.1$, and 0.6 denoted by the light blue, orange, and red slopes, accordingly. The dark blue line depicts the mRNA Fano factor when an extrinsic factor is not included. Monte Carlo Simulations (SSA) results are denoted by circle markers and validate the analytical formulas. The remaining parameters have the following values: $\langle B\rangle=10, k_{x}=10,\left\langle B_{z}\right\rangle=10, \gamma_{z}=1$, $t_{x}=1$. 
time is ideal as it offers better mRNA noise buffering, while increased RNA transport time increases mRNA noise.

We further explore the extent of the extrinsic factor dynamics on shaping mRNA noise by considering the extrinsic species time scale. We again find interesting nonmonotonic behavior in the mRNA Fano factor, depicted in Fig. 3, where noise is plotted as a function of the extrinsic factor decay rate $\left(\gamma_{z}\right)$. The mRNA Fano factor increases at low extrinsic factor time scales. However, as the timescale is increased, the mRNA noise is buffered. We additionally find that when the extrinsic factor has increased variability, the mRNA Fano factor is also increased.

\subsubsection{Extrinsic Fluctuations for a Multi-Step Time Delayed Export}

Extrinsic disturbances are next incorporated into a time delayed nuclear export process. Here, the export process is modeled as multiple events, implemented through irreversible conversion reactions converting the nuclear RNA $X_{0}$ into cytoplasmic mRNA $X_{n}$ by intermediate states: $X_{1}, \ldots X_{n-1}$, where the rate of conversion is given by $n z(t) t_{x} / \overline{\langle z\rangle}$. Note that for a fixed $z$, this corresponds to an Erlang-distributed nuclear retention time. For a two-step export process the overall stochastic model is illustrated in Table II

Table 2: Stochastic gene expression model of a two-step nuclear export process $(n=2$; the incorporation of extrinsic noise species $Z$ is described under the the dashed line. Here $x_{i}(t)$ denotes the population count of species $X_{i}$ at time $t$.

\begin{tabular}{|c|c|c|}
\hline Event & $\begin{array}{c}\text { Change in } \\
\text { Population Count }\end{array}$ & $\begin{array}{l}\text { Probability Event will } \\
\text { Occur in }(t, t+d t)\end{array}$ \\
\hline Transcriptional Bursting & $x_{0}(t) \rightarrow x_{0}(t)+j$ & $k_{x} \alpha_{j} d t, j=1,2, .$. \\
\hline Nuclear Export & $\begin{array}{l}x_{0}(t) \rightarrow x_{0}(t)-1 \\
x_{1}(t) \rightarrow x_{1}(t)+1\end{array}$ & $\frac{2 t_{x} z(t)}{\langle z\rangle} x_{0}(t) d t$ \\
\hline Nuclear Export & $\begin{array}{l}x_{1}(t) \rightarrow x_{1}(t)-1 \\
x_{2}(t) \rightarrow x_{2}(t)+1\end{array}$ & $\frac{2 t_{x} z(t)}{\langle z\rangle} x_{1}(t) d t$ \\
\hline mRNA Degradation & $x_{2}(t) \rightarrow x_{2}(t)-1$ & $\gamma_{x} x_{2}(t) d t$ \\
\hline Extrinsic Noise Production & $z(t) \rightarrow z(t)+i$ & $\begin{array}{c}k_{z} \alpha_{i} d t, i=1,2, . . \\
\end{array}$ \\
\hline Extrinsic Noise Degradation & $z(t) \rightarrow z(t)-1$ & $\gamma_{z} z(t) d t$ \\
\hline
\end{tabular}

Using the Method of Moments, we derive the following formula for the steady-state mRNA Fano factor corresponding to a two-step time delay $(n=2)$ in the presence of an extrinsic disturbance 


$$
F F_{n=2}=1+\frac{\left\langle B^{2}\right\rangle-\langle B\rangle}{\langle B\rangle} \frac{t_{x}\left(\gamma_{x}+4 t_{x}\right)}{2\left(\gamma_{x}+2 t_{x}\right)^{2}}+C V_{Z}^{2} \frac{\langle B\rangle \gamma_{x} \gamma_{z} k_{x}\left(\gamma_{x} \gamma_{z}+5\left(\gamma_{x}+\gamma_{z}\right) t_{x}+16 t_{x}^{2}\right)}{\left(\gamma_{x}+\gamma_{z}\right)\left(\gamma_{z}+2 t_{x}\right)^{2}\left(\gamma_{x}+2 t_{x}\right)^{2}}
$$

Note by taking $C V_{Z}^{2}=0$ one would obtain the same Fano factor as obtained using (3) for an Erlang-distributed nuclear retention time.

Using a similar process the Fano factor can be derived for a three-step time-delayed export process $(n=3)$. Results for the one-step and three-step transport process are compared in Fig. 4. The Fano factor is plotted as a function of increasing average RNA transport time and is shown for when the extrinsic factor is, and is not included. First, we note that when the one-step and three-step transport processes are not in the presence of extrinsic fluctuations, the mRNA noise is similar. The mRNA noise becomes the same at increased average RNA transport time. Next, we examine the one-step and three-step transport processes when the extrinsic factor is included. The mRNA Fano factor exhibits greater noise when extrinsic fluctuations are included in the three-step process compared to the one-step process. Additionally, when nuclear export has a one-step or three-step delay, the Fano factor exhibits non-monotonic behavior, where the non-monotonic behavior is more apparent in the three-step delay. Last, when average RNA transport time is increased, the Fano factor for the one-step and three-step delay eventually results in the same mRNA Fano factor.

\section{Conclusion}

We have systematically studied the role that different characteristics of the nuclear export process play in shaping the extent of random fluctuations in the mRNA level of a given gene. We considered how mRNA noise is affected by different nuclear retention time distributions, by time-delayed nuclear export, and by extrinsic fluctuations in nuclear export. In regards to nuclear export timing, a general observation found is that increased average export time, thus a small export rate buffers cytoplasmic mRNA noise.

Specifically, there are regimes where increased variability in RNA transport time actually buffers mRNA noise. Additionally, when extrinsic fluctuations are incorporated, mRNA noise exhibits non-monotonic behavior when plotted as a function of extrinsic species timescale, where noise is originally low for stable extrinsic species, then sharply increases as extrinsic species stability is decreased, before decreasing again for extremely unstable extrinsic species. Furthermore, the effects of extrinsic fluctuations are magnified when the fluctuations are incorporated into $n$-step delayed nuclear export.

\section{A Fano Factor using Partitioning of Poisson Arrivals Method}

We apply the mapping based on partitioning of Poisson arrivals (PPA-mapping) [30] to consider a reduced model with bursts arriving at rate $k_{x} / N$ where $N \rightarrow \infty$. There- 


\section{Noise in mRNA level when extrinsic disturbance affects three-step nuclear export process}

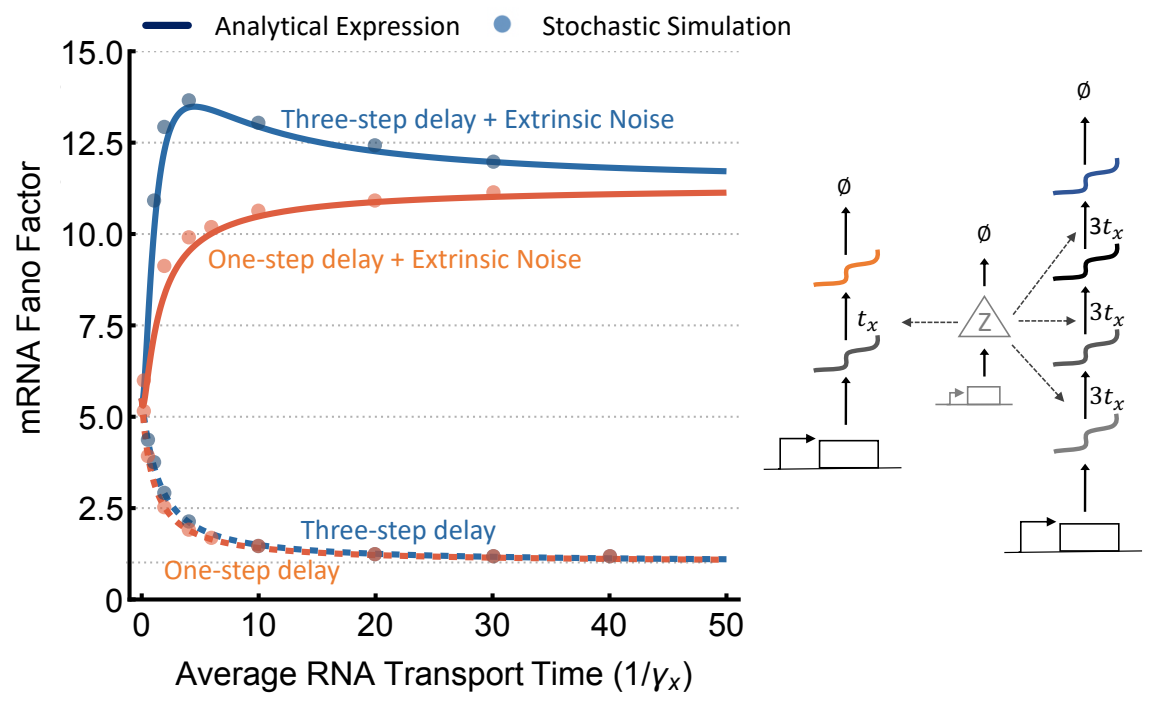

Figure 4: Cytoplasmic mRNA level noise plotted as a function of increasing nuclear RNA transport time, normalized by the mRNA decay rate, comparing time delayed nuclear export with and without the presence of extrinsic noise, denoted by solid and dashed lines, accordingly. The three-step export process is denoted by blue and the one-step delay is denoted by orange. The analytical formulas (lines) are validated by Monte Carlo Simulations (SSA), denoted by the circle markers. The remaining parameters have the following values: $\langle B\rangle=10, k_{x}=10, k_{z}=4$, and $\gamma_{z}=3$. 
fore, the mRNA generating functions for the the original $G(y, t)$ and the reduced $g(y, t)$ models are related by

$$
G(y, t)=\lim _{N \rightarrow \infty} \exp [N(g(y, t)-1)] .
$$

In the next step we derive $g(y, t)$. For the reduced model, bursts are arriving with rate $k_{x} / N$, i.e., the number of burst arrivals at time $t$ is a Poisson-distributed random variable with mean $k_{x} t / N$. In the limit of $N \rightarrow \infty$, we only need to consider the 0-burst and 1-burst arrival cases in time $t$. Correspondingly, we have

$$
\begin{aligned}
g(y, t) & =\overbrace{e^{-\frac{k_{x} t}{N}}}^{\text {Prob.of 0 burst }}+\overbrace{\left(1-e^{-\frac{k_{x} t}{N}}\right) g_{1}(y, t)}^{\text {Prob. of burst }>0} \\
\Rightarrow g(y, t) & =1-\frac{k_{x} t}{N}+\frac{k_{x} t}{N} g_{1}(y, t),
\end{aligned}
$$

where $g_{1}(y, t)$ is the generating function of mRNA distribution at time $t$, conditional on exactly 1 burst arrival between time 0 to $t$. To this end, in order to calculate $G(y, t)$ we need to calculate $g_{1}(y, t)$.

Consider a single mRNA is created at $t=s$. Conditioning on the export time $\left(s_{1}\right)$, the generating function for the distribution of mRNAs at time $t$ is given by

$$
\begin{gathered}
f_{1}(y, t-s)=y \int_{s}^{t} h\left(s_{1}-s\right) e^{-\gamma_{x}\left(t-s_{1}\right)} \\
+\left[1-\int_{s}^{t} h\left(s_{1}-s\right) e^{-\gamma_{x}\left(t-s_{1}\right)}\right]
\end{gathered}
$$

Let $g_{b}(y)$ be the generating function for probability distribution of number of RNAs produced in the burst. Then, by conditioning on the time of burst arrival $t=s$ we have

$$
g_{1}(y, t)=\int_{0}^{t} \frac{d s}{t} \underbrace{g_{b}(\overbrace{f_{1}(y, t-s)}^{\text {single mRNA }})}_{\text {contribution of the entire burst }}
$$

The above expression is conditioned on having a single bursting event which means the burst arrival time distribution is uniform over $[0, t]$. Substituting (13) in (11), and then using (10) we get

$$
G(y, t)=\lim _{N \rightarrow \infty} \exp \left[N\left(1-\frac{k_{x} t}{N}+\frac{k_{x} t}{N} \int_{0}^{t} \frac{1}{t} g_{b}\left(f_{1}(y, t-s)\right) d s-1\right)\right]
$$

simplifying to

$$
G(y, t)=\exp \left[k_{x} \int_{0}^{t}\left(g_{b}\left(f_{1}(y, t-s)\right)-1\right)\right] .
$$

By having the generating function we can calculate the mean and variance of the mRNA counts $x_{1}(t)$ by using the following formulas

$$
\left.G^{\prime}(y)\right|_{y=1}=\left\langle x_{1}\right\rangle,\left.G^{\prime \prime}(y)\right|_{y=1}=\left\langle x_{1}\left(x_{1}-1\right)\right\rangle .
$$


Hence, we can derive the mean count as

$$
\begin{aligned}
\left\langle x_{1}\right\rangle= & \left.g_{b}^{\prime}(y)\right|_{y=1} \times \\
& k_{x} \int_{0}^{t}\left[\int_{s}^{t} h\left(s_{1}-s\right) e^{-\gamma_{x}\left(t-s_{1}\right)} d s_{1}\right] d s,
\end{aligned}
$$

and its second-order moment as

$$
\left\langle x_{1}\left(x_{1}-1\right)\right\rangle=\left\langle x_{1}\right\rangle^{2}+g_{b}^{\prime \prime}(y) \times\left.\int_{0}^{t}\left(f_{1}^{\prime}(y, t-s)\right)^{2} d s\right|_{y=1} .
$$

By replacing the burst size's first- and second-order moments as

$$
\left.g_{b}^{\prime}(y)\right|_{y=1}=\langle B\rangle,\left.\quad g_{b}^{\prime \prime}(y)\right|_{y=1}=\langle B(B-1)\rangle,
$$

we have

$$
F F=1+\frac{\left\langle B^{2}\right\rangle-\langle B\rangle}{\langle B\rangle} \frac{\int_{0}^{t}\left[\int_{s}^{t} h\left(s_{1}-s\right) e^{-\gamma_{x}\left(t-s_{1}\right)} d s_{1}\right]^{2} d s}{\int_{0}^{t}\left[\int_{s}^{t} h\left(s_{1}-s\right) e^{-\gamma_{x}\left(t-s_{1}\right)} d s_{1}\right] d s}
$$

Let $\tau$ denote the mean waiting time for mRNA decay including RNA export time. Then by using Little's law we have

$$
\left\langle x_{1}\right\rangle=k_{x}\langle B\rangle \tau
$$

Hence from (17) we have

$$
\tau=\int_{0}^{t}\left[\int_{s}^{t} h\left(s_{1}-s\right) e^{-\gamma_{x}\left(t-s_{1}\right)} d s_{1}\right] d s .
$$

\section{B Fano Factor using Method of Moments}

To characterize the effects of extrinsic noise in the nuclear export rate, we again obtain the Fano factor. To include the extrinsic noise species, $Z$, we utilize the Method of Moments to obtain the Fano factor of the export model described by the stochastic formulation described in Table 1. In this table, the extrinsic noise factor is described in the rows below the dashed line. We assume each event is probabilistic and occurring at exponentially-distributed time intervals. When an event occurs, the nuclear RNA and cytoplasmic mRNA population count must be updated, as described by the resets in the center column of the table. The propensity functions in the rightmost column describe the probability that the event will occur in the next infinitesimal time. The time derivative of the expected value of any differentiable function $\varphi\left(x_{0}, x_{1}, z\right)$ is given 
by [37]:

$$
\begin{aligned}
& \frac{d\left\langle\varphi\left(x_{0}, x_{1}, z\right)\right\rangle}{d t}= \\
& \left\langle\sum_{B=1}^{\infty} \alpha_{B} k_{x}\left[\varphi\left(x_{0}+B, x_{1}, z\right)-\varphi\left(x_{0}, x_{1}, z\right)\right]\right. \\
& +\sum_{B_{z}=1}^{\infty} \alpha_{B_{z}} k_{z}\left[\varphi\left(x_{0}, x_{1}, z+B_{z}\right)-\varphi\left(x_{0}, x_{1}, z\right)\right] \\
& \quad+t_{x} x_{0} \frac{z}{\langle\bar{z}\rangle}\left[\varphi\left(x_{0}-1, x_{1}+1, z\right)-\varphi\left(x_{0}, x_{1}, z\right)\right] \\
& \left.\quad+\gamma_{x} x_{1}\left[\varphi\left(x_{0}, x_{1}-1\right), z\right)-\varphi\left(x_{0}, x_{1}, z\right)\right] \\
& \left.\quad+\gamma_{z} z\left[\varphi\left(x_{0}, x_{1}, z-1\right)-\varphi\left(x_{0}, x_{1}, z\right)\right]\right\rangle .
\end{aligned}
$$

The Fano factor is found by first selecting appropriate choices for the function $\varphi\left(x_{0}, x_{1}, z\right)$ to obtain the first- and second- order moments [37]. We then solve for steady-state to determine the mean and variance of mRNA level. In this model, nonlinearity is introduced in the export rate which takes the form $t_{x} z x_{0}$. The non-linearity causes the system to become analytically intractable. To obtain the Fano factor we linearize this rate using Taylor Series expansion. We assume small fluctuations in nuclear RNA count $x_{0}(t)$ and extrinsic noise factor count $z(t)$ [38-40]. By linearizing the transport rate about its respective means $\overline{\left\langle x_{0}\right\rangle}$ and $\overline{\langle z\rangle}$, we approximate the following transport rate as

$$
x_{0} z \approx \overline{\left\langle x_{0}\right\rangle\langle z\rangle}+\overline{\langle z\rangle}\left(x_{0}-\overline{\left\langle x_{0}\right\rangle}\right)+\overline{\left\langle x_{0}\right\rangle}(z-\overline{\langle z\rangle}) .
$$

When obtaining the moment dynamics, we replace the non-linear transport rate in (23) with the linear rate (24).

Selecting appropriate choices for $\varphi\left(x_{0}, x_{1}, z\right)$ and using the resets and propensity 
functions described in Table I, we reveal the following moment dynamics:

$$
\begin{aligned}
& \frac{d\left\langle x_{0}\right\rangle}{d t}=\langle B\rangle k_{x}-t_{x}\left\langle x_{0}\right\rangle+t_{x} \overline{\left\langle x_{0}\right\rangle}-\frac{t_{x} \overline{\left\langle x_{0}\right\rangle}\langle z\rangle}{\overline{\langle z\rangle}} \\
& \frac{d\left\langle x_{1}\right\rangle}{d t}=t_{x}\left\langle x_{0}\right\rangle-t_{x} \overline{\left\langle x_{0}\right\rangle}-\gamma_{x} x_{1}+\frac{t_{x} \overline{\left\langle x_{0}\right\rangle}\langle z\rangle}{\overline{\langle z\rangle}} \\
& \frac{d\langle z\rangle}{d t}=\left\langle B_{z}\right\rangle k_{z}-\gamma_{z}\langle z\rangle \\
& \frac{d\left\langle x_{0}^{2}\right\rangle}{d t}=\left\langle B^{2}\right\rangle k_{x}+2\langle B\rangle k_{x}\left\langle x_{0}\right\rangle+t_{x}\left\langle x_{0}\right\rangle-2 t_{x}\left\langle x_{0}^{2}\right\rangle-t_{x} \overline{\left\langle x_{0}\right\rangle} \\
& +2 t_{x}\left\langle x_{0}\right\rangle \overline{\left\langle x_{0}\right\rangle}+\frac{t_{x} \overline{\left\langle x_{0}\right\rangle}\langle z\rangle}{\overline{\langle z\rangle}}+\frac{2 t_{x} \overline{\left\langle x_{0}\right\rangle}\left\langle x_{0}\right\rangle\langle z\rangle}{\overline{\langle z\rangle}} \\
& \frac{d\left\langle x_{1}^{2}\right\rangle}{d t}=t_{x}\left\langle x_{0}\right\rangle-t_{x} \overline{\left\langle x_{0}\right\rangle}+\gamma_{x}\left\langle x_{1}\right\rangle+2 t_{x}\left\langle x_{0}\right\rangle\left\langle x_{1}\right\rangle-2 t_{x} \overline{\left\langle x_{0}\right\rangle}\left\langle x_{1}\right\rangle \\
& -2 \gamma_{x}\left\langle x_{1}^{2}\right\rangle+\frac{t_{x} \overline{\left\langle x_{0}\right\rangle}\langle z\rangle}{\overline{\langle z\rangle}}+\frac{2 t_{x} \overline{\left\langle x_{0}\right\rangle}\left\langle x_{1}\right\rangle\langle z\rangle}{\overline{\langle z\rangle}} \\
& \frac{d\left\langle z^{2}\right\rangle}{d t}=\left\langle B_{z}^{2}\right\rangle k_{z}+\gamma_{z}\langle z\rangle+2\left\langle B_{z}\right\rangle k_{z}\langle z\rangle-2 \gamma_{z}\left\langle z^{2}\right\rangle \\
& \frac{d\left\langle x_{0} x_{1}\right\rangle}{d t}=-t_{x}\left\langle x_{0}\right\rangle+t_{x}\left\langle x_{0}^{2}\right\rangle+t_{x} \overline{\left\langle x_{0}\right\rangle}-t_{x}\left\langle x_{0}\right\rangle \overline{\left\langle x_{0}\right\rangle}+\langle B\rangle k_{x}\left\langle x_{1}\right\rangle \\
& -\gamma_{x}\left\langle x_{0}\right\rangle\left\langle x_{1}\right\rangle-t_{x}\left\langle x_{0}\right\rangle\left\langle x_{1}\right\rangle+t_{x} \overline{\left\langle x_{0}\right\rangle}\left\langle x_{1}\right\rangle \\
& -\frac{t_{x} \overline{\left\langle x_{0}\right\rangle}\langle z\rangle}{\overline{\langle z\rangle}}+\frac{t_{x} \overline{\left\langle x_{0}\right\rangle}\left\langle x_{0}\right\rangle\langle z\rangle}{\overline{\langle z\rangle}}-\frac{t_{x} \overline{\left\langle x_{0}\right\rangle}\left\langle x_{1}\right\rangle\langle z\rangle}{\overline{\langle z\rangle}} \\
& \frac{d\left\langle x_{0} z\right\rangle}{d t}=\left\langle B_{z}\right\rangle k_{z}\left\langle x_{0}\right\rangle+\langle B\rangle k_{x}\langle z\rangle-\gamma_{z}\left\langle x_{0}\right\rangle\langle z\rangle-t_{x}\left\langle x_{0}\right\rangle\langle z\rangle \\
& +t_{x} \overline{\left\langle x_{0}\right\rangle}\langle z\rangle-\frac{t_{x} \overline{\left\langle x_{0}\right\rangle}\left\langle z^{2}\right\rangle}{\overline{\langle z\rangle}} \\
& \frac{d\left\langle x_{1} z\right\rangle}{d t}=\left\langle B_{z}\right\rangle k_{z}\left\langle x_{1}\right\rangle+t_{x}\left\langle x_{0}\right\rangle\langle z\rangle-t_{x} \overline{\left\langle x_{0}\right\rangle}\langle z\rangle-\gamma_{x}\left\langle x_{1}\right\rangle\langle z\rangle \\
& -\gamma_{z}\left\langle x_{1}\right\rangle\langle z\rangle+\frac{t_{x} \overline{\left\langle x_{0}\right\rangle}\left\langle z^{2}\right\rangle}{\overline{\langle z\rangle}}
\end{aligned}
$$

$[41,42]$. Quantifying the steady-state first- and second-order moments results in the Fano factor (7).

\section{ACKNOWLEDGMENT}

This work is supported by the NSF grant ECCS-1711548 and ARO grant W911NF1910243 to AS, and NSF grant DMS-1854350 to RVK. 


\section{References}

[1] W. J. Blake, M. Kaern, C. R. Cantor, and J. J. Collins, "Noise in eukaryotic gene expression," Nature, vol. 422, pp. 633-637, 2003.

[2] J. M. Raser and E. K. O'Shea, "Noise in gene expression: Origins, consequences, and control," Science, vol. 309, pp. 2010 - 2013, 2005.

[3] E. Libby, T. J. Perkins, and P. S. Swain, "Noisy information processing through transcriptional regulation," Proceedings of the National Academy of Sciences, vol. 104, pp. 7151-7156, 2007.

[4] B. Lehner, "Selection to minimise noise in living systems and its implications for the evolution of gene expression," Molecular Systems Biology, vol. 4, p. 170, 2008.

[5] H. B. Fraser, A. E. Hirsh, G. Giaever, J. Kumm, and M. B. Eisen, "Noise minimization in eukaryotic gene expression," PLOS Biology, vol. 2, p. e137, 2004.

[6] I. Golding, J. Paulsson, S. Zawilski, and E. Cox, "Real-time kinetics of gene activity in individual bacteria," Cell, vol. 123, pp. 1025-1036, 2005.

[7] A. Bar-Even, J. Paulsson, N. Maheshri, M. Carmi, E. O'Shea, Y. Pilpel, and N. Barkai, "Noise in protein expression scales with natural protein abundance," Nature Genetics, vol. 38, pp. 636-643, 2006.

[8] A. Eldar and M. B. Elowitz, "Functional roles for noise in genetic circuits," Nature, vol. 467, pp. 167-173, 2010.

[9] M. B. Elowitz, A. J. Levine, E. D. Siggia, and P. S. Swain, "Stochastic gene expression in a single cell," Science, vol. 297, pp. 1183-1186, 2002.

[10] Y. Taniguchi, P. J. Choi, G. W. Li, H. Chen, M. Babu, J. Hearn, A. Emili, and X. S. Xie, "Quantifying E. coli proteome and transcriptome with single-molecule sensitivity in single cells," Science, vol. 329, pp. 533-538, 2010.

[11] J. R. S. Newman, S. Ghaemmaghami, J. Ihmels, D. K. Breslow, M. Noble, J. L. DeRisi, and J. S. Weissman, "Single-cell proteomic analysis of S. cerevisiae reveals the architecture of biological noise," Nature Genetics, vol. 441, pp. 840846, 2006.

[12] P. S. Swain, M. B. Elowitz, and E. D. Siggia, "Intrinsic and extrinsic contributions to stochasticity in gene expression," Proceedings of the National Academy of Sciences, vol. 99, pp. 12 795-12 800, 2002.

[13] A. Singh and M. Soltani, "Quantifying intrinsic and extrinsic variability in stochastic gene expression models," PLOS ONE, vol. 8, p. e84301, 2013.

[14] M. Scott, B. Ingalls, and M. Kaern, "Estimations of intrinsic and extrinsic noise in models of nonlinear genetic networks," CHAOS, vol. 16, p. 026107, 2006. 
[15] D. Orrell and H. Bolouri, "Control of internal and external noise in genetic regulatory networks," Journal of Theoretical Biology, vol. 230, pp. 301-312, 2004.

[16] A. Hilfinger and J. Paulsson, "Separating intrinsic from extrinsic fluctuations in dynamic biological systems," Proceedings of the National Academy of Sciences, vol. 108, pp. 12 167-12 172, 2011.

[17] I. G. Johnston, B. Gaal, R. P. das Neves, T. Enver, F. J. Iborra, and N. S. Jones, "Mitochondrial variability as a source of extrinsic cellular noise," PLOS Computational Biology, vol. 8, p. e1002416, 2012.

[18] C. J. Zopf, K. Quinn, J. Zeidman, and N. Maheshri, "Cell-cycle dependence of transcription dominates noise in gene expression," PLOS Computational Biology, vol. 9, p. e1003161, 2013.

[19] O. Padovan-Merhar, G. P. Nair, A. G. Biaesch, A. Mayer, S. Scarfone, S. W. Foley, A. R. Wu, L. S. Churchman, A. Singh, and A. Raj, "Single mammalian cells compensate for differences in cellular volume and DNA copy number through independent global transcriptional mechanisms," Molecular Cell, vol. 58, pp. 339$352,2015$.

[20] M. Okamura, H. Inose, and S. Masuda, "Rna export through the npc in eukaryotes." Genes (Basel), vol. 6, pp. 124-149, 2015.

[21] S. R. Carmody and S. R. Wente, "mrna nuclear export at a glance," Journal of Cell Science, vol. 122, pp. 933-1937, 2009.

[22] G. Gorin and L. Pachter, "Special function methods for bursty models of transcription," Phys. Rev. E, vol. 102, p. 022409, Aug 2020. [Online]. Available: https://link.aps.org/doi/10.1103/PhysRevE.102.022409

[23] R. D. Dar, S. M. Shaffer, A. Singh, B. S. Razooky, M. L. Simpson, A. Raj, and L. S. Weinberger, "Transcriptional bursting explains the noise-versus-mean relationship in mRNA and protein levels," PLOS ONE, vol. 11, p. e0158298, 2016.

[24] A. Singh, B. S. Razooky, R. D. Dar, and L. S. Weinberger, "Dynamics of protein noise can distinguish between alternate sources of gene-expression variability," Molecular Systems Biology, vol. 8, p. 607, 2012.

[25] A. Raj, C. Peskin, D. Tranchina, D. Vargas, and S. Tyagi, "Stochastic mRNA synthesis in mammalian cells," PLOS Biology, vol. 4, p. e309, 2006.

[26] R. D. Dar, B. S. Razooky, A. Singh, T. V. Trimeloni, J. M. McCollum, C. D. Cox, M. L. Simpson, and L. S. Weinberger, "Transcriptional burst frequency and burst size are equally modulated across the human genome," Proceedings of the National Academy of Sciences, vol. 109, pp. 17 454-17459, 2012.

[27] T. Jia and R. V. Kulkarni, "Intrinsic noise in stochastic models of gene expression with molecular memory and bursting," Journal of Mathematical Biology, vol. 106, p. 058102, 2011. 
[28] N. Kumar, A. Singh, and R. V. Kulkarni, "Transcriptional bursting in gene expression: Analytical results for genera stochastic models," PLOS Computational Biology, vol. 11, p. e1004292, 2015.

[29] S. Modi, S. Dey, and A. Singh, "Noise suppression in stochastic genetic circuits using pid controllers," PLoS Computational Biology, vol. 17, p. e1009249, 2021.

[30] H. Pendar, T. Platini, and R. V. Kulkarni, "Exact protein distributions for stochastic models of gene expression using partitioning of poisson processes," Phys. Rev. E, vol. 87, p. 042720, 2013.

[31] A. Singh and P. Bokes, "Consequences of mRNA transport on stochastic variability in protein levels," Biophysical Journal, vol. 103, pp. 1087-1096, 2012.

[32] N. Battich, T. Stoeger, and L. Pelkmans, "Control of transcript variability in single mammalian cells," Cell, vol. 163, pp. 1596-1610, 2015.

[33] K. B. Halpern, I. Caspi, D. Lemze, M. Levy, S. Landen, E. Elinav, I. Ulitsky, and S. Itzkovitz, "Nuclear retention of mrna in mammalian tissues," Cell reports, vol. 13, pp. 2653-2662, 2015.

[34] T. Stoeger, N. Battich, and L. Pelkmans, "Passive noise filtering by cellular compartmentalization," Cell, vol. 164, pp. 1151-1161, 2016.

[35] M. M. Hansen, R. V. Desai, M. L. Simpson, and L. S. Weinberger, "Cytoplasmic amplification of transcriptional noise generates substantial cell-to-cell variability," Cell systems, vol. 7, pp. 384-397, 2018.

[36] Q. Wang and T. Zhou, "Alternative-splicing-mediated gene expression," Physical Review E, vol. 89, p. 012713, 2014.

[37] A. Singh and J. P. Hespanha, "Stochastic hybrid systems for studying biochemical processes," Philosophical Transactions of the Royal Society A, vol. 368, pp. 4995-5011, 2010.

[38] J. Elf and M. Ehrenberg, "Fast evaluation of fluctuations in biochemical networks with the linear noise approximation." Genome Research, vol. 13, pp. 2475-2484, 2003.

[39] N. Van Kampen, Stochastic Processes in Physics and Chemistry. Elsevier, 2011.

[40] S. Modi, M. Soltani, and A. Singh, "Linear noise approximation for a class of piecewise deterministic markov processes," in 2018 Annual American Control Conference (ACC), 2018, pp. 1993-1998.

[41] J. P. Hespanha and A. Singh, "Stochastic models for chemically reacting systems using polynomial stochastic hybrid systems," International Journal of Robust and Nonlinear Control, vol. 15, pp. 669-689, 2005.

[42] A. Singh and J. P. Hespanha, "Approximate moment dynamics for chemically reacting systems," IEEE Transactions on Automatic Control, vol. 56, pp. 414418, 2011. 